\title{
Associations Between Cocaine, Amphetamine or Psychedelic Use and Psychotic Symptoms in a Community Sample
}

Nina Kuzenko, M.D. ${ }^{1}$, Jitender Sareen, M.D. ${ }^{1 *}$, Katja Beesdo-Baum, Ph.D. ${ }^{2}$, Axel Perkonigg, Ph.D. ${ }^{2}$, Michael Höfler, Ph.D. ${ }^{2}$, James Simm, M.D. ${ }^{1}$, Roselind Lieb, Ph.D. ${ }^{3,4}$, Hans-Ulrich Wittchen, Ph.D. ${ }^{2,4}$

${ }^{1}$ Department of Psychiatry, University of Manitoba, Winnipeg, Manitoba, Canada

${ }^{2}$ Institute of Clinical Psychology and Psychotherapy, Technische Universitaet Dresden, Dresden, Germany

${ }^{3}$ Institute of Psychology, Epidemiology and Health Psychology, University of Basel, Basel, Switzerland

${ }^{4}$ Max-Planck-Institute of Psychiatry, Munich, Germany

*Corresponding Author: J. Sareen, Associate Professor of Psychiatry, Psychology and Community Health PZ-430 771 Bannatyne Avenue, Winnipeg, Manitoba, Canada, R3E 3N4, 204-787-7078, sareen@cc.umanitoba.ca 
Objective: To investigate whether there is an association between use of cocaine, amphetamines, or psychedelics and psychotic symptoms.

Method: Cumulated data from a prospective, longitudinal community study of 2588 adolescents and young adults in Munich, Germany were used. Substance use was assessed at baseline, 4year and 10-year follow-up using the Munich Composite International Diagnostic Interview; psychotic symptoms were assessed at 4-year and 10-year follow-up. Multinomial logistic regression analyses, adjusted for sociodemographic factors, common mental disorders, other substance use, and childhood adversity (adjusted odds ratios, AOR), revealed associations between cocaine, amphetamine or psychedelic use and psychotic symptoms.

Results: Lifetime experience of psychotic symptoms was associated with lifetime use of cocaine (AOR 1.94; 95\%CI 1.10-3.45), amphetamines (AOR 1.69; 95\%CI 0.98-2.93), psychedelics (AOR 2.37; 95\%CI 1.20-4.66) and all three substances (AOR 1.95; 95\%CI 1.193.18).

Conclusion: Associations between psychotic symptoms and use of cocaine, amphetamines, and/or psychedelics in adolescents and young adults call for further studies to elucidate risk factors and developmental pathways.

Keywords: Substance-Related Disorders, Cocaine, Amphetamine, Psychedelics, Psychoses 


\section{Significant Outcomes}

- After adjusting for potential confounding factors, use of cocaine, amphetamines or psychedelics was associated with psychotic symptoms.

- Among a subgroup of persons without a mood or anxiety disorder, analysis revealed an association between use of cocaine, amphetamines or psychedelics and psychotic symptoms.

\section{Limitations}

- The low positive predictive value of the M-CIDI does not allow for analysis of psychotic disorders; analysis is limited to psychotic symptoms.

- The relatively low prevalence of psychotic symptoms among this community sample limited the ability to analyse for a temporal relationship between substance use and psychotic symptoms.

- Self-report of lifetime substance use and psychotic symptoms may lead to recall errors or erroneous information. 


\section{Introduction}

Although the association of drug use with mood, anxiety and personality disorders has been well documented (1-3), there has been an increased interest in the risk of psychosis among those using illegal drugs. Several clinical and community-based studies have demonstrated an association between cannabis use and psychotic symptoms (4-11). Non-cannabis drug use has been examined among persons with established psychotic disorders $(12,13)$ and psychotic symptoms (14-17). Some of these studies demonstrate an association between use of stimulants and psychotic symptoms $(12,15-17)$. In investigating the temporal relationship between substance (e.g. cannabis, amphetamine, cocaine, psychedelics) use and onset of psychotic symptoms, several studies report that in the majority of study participants, substance use was initiated prior to experiencing psychotic symptoms (17-20). However, there are also findings referring to predominantly secondary onset of substance use after psychotic symptoms (21). In the present study, we aim to extend this area of inquiry by examining the association between use of three non-cannabinoid illegal drugs - cocaine, amphetamines and psychedelics and psychotic symptoms. We chose these specific illegal drugs because each of them has neurobiological underpinnings to psychosis. For example, it has long been known that cocaine and amphetamines increase levels of dopamine by powerfully blocking the dopamine transporter (22-24). While psychedelics are primarily serotonin-2A receptor agonists, lysergic acid diethylamide (LSD) in particular is known to have a high affinity for dopamine receptors $(25,26)$. It is not surprising, then, that substance intoxication with these substances includes psychotic symptoms such as paranoia and hallucinations. 
Although elevated levels of psychotic symptoms among persons using cocaine or amphetamines have been reported in general population samples $(14,16)$, clinical samples $(12,13)$ and among prison inmates $(15)$, the strength of the association has varied considerably.

There are a number of limitations of studies on non-cannabinoid illegal drug use and psychotic symptoms. First, studies using clinical or forensic samples are limited by selection bias $(12,13,15)$. Second, many studies have not adjusted for confounding variables such as other mental disorders, drug-induced psychosis, and use of cannabis or other drugs, such as opiates, sedatives, or PCP $(13,14,16,17)$.

Using data from a prospective-longitudinal community study of adolescents and young adults, we examine associations between cocaine, amphetamine or psychedelic use and psychotic symptoms. Since psychotic symptoms can occur during the intoxication phase of these noncannabinoid drugs, we were specifically interested in examining whether these drugs were associated with psychotic symptoms that were not limited to the intoxication periods. We predict that cocaine, amphetamine or psychedelic use will be associated with psychotic symptoms, independent of sociodemographic factors, adverse childhood experiences, and other drug (cannabis, opiates, sedatives, PCP) use, which are factors known to be associated with development of psychotic symptoms $(27,28)$. Due to methodological limitations, we were unable to examine temporal relationships between substance use and psychotic symptoms in this study.

\section{Aims of the study}

To examine the association between use of cocaine, amphetamine and psychedelics and psychotic symptoms in a large, representative community sample of adolescents and young adults followed over approximately 10 years. 


\section{Methods}

\subsection{Sample and overall design}

Data were collected as part of the Early Developmental Stages of Psychopathology (EDSP) study. The EDSP is a prospective-longitudinal study on prevalence, incidence, risk factors and course of substance use disorders and mental disorders in a representative community sample of adolescents and young adults $(29,30)$. The baseline investigation was conducted in 1995 with the total sample of 14 - to 24 -year-olds $(\mathrm{N}=3,021)$ and a response rate of $71 \%$. The first follow-up study was conducted only among the younger cohort in 1996/1997 with a response rate of $88 \%$ among 14-17 year olds. The second follow-up (“4-year follow-up”) was carried out in 1998/1999 with a response rate of $84 \%$ among all 14- to 24 -year-olds of the baseline investigation. The third follow-up ("10-year follow-up") was also conducted among all participants in 2004/2005 and reached a response rate of $73 \%$.

The sample was randomly drawn from government registries in Munich, Germany. All participants provided informed consent after complete description of the study. Because the study was designed as a longitudinal panel with emphasis on the early developmental stages of psychopathology and substance use disorders, 14-15-year-olds were sampled at twice the probability of 16-21-year-olds, and four times the probability of 22-24-year-olds. The community sample has been demonstrated to be representative of the general population (29).

\subsection{Diagnostic Assessment}

Diagnostic assessments in all waves were based on the computer-assisted MunichComposite International Diagnostic Interview (M-CIDI/DIA-X, 31) which allows for the assessment of symptoms, syndromes, and diagnoses of 48 mental disorders according to the 
DSM-IV criteria (32) and for collection of data on onset, duration, severity, and psychosocial impairment. Diagnostic findings were obtained by using the M-CIDI/DSM-IV algorithms. At baseline, the lifetime version of the M-CIDI was used. At each follow-up, the interval version was applied. In all assessments, the M-CIDI was supplemented by a respondents' booklet that included scales and questionnaires for assessing psychological constructs relevant to the study (30). Interviews were conducted by trained clinical interviewers (mainly psychologists in postgraduate training) and closely monitored by the staff members and clinical supervisors during weekly supervision sessions. Correct interview administration was also verified by phonecalls to participants. Follow-up interviews were conducted using the same procedures $(29,30)$. Test-retest reliability and validity, which were fair to good (kappa 0.56-0.81 for DSM-IV diagnostic categories), have been reported in detail elsewhere $(33,34)$.

\subsubsection{Assessment of substance use and substance use disorders}

The methods and previous results on substance use disorders of the study have been published in greater detail elsewhere (1,35-43). Briefly, cocaine, amphetamines, and psychedelics and other illegal substance use (frequency and quantity), as well as DSM-IV abuse and dependence were assessed in Section L (drugs) of the M-CIDI/DIA-X. The section starts with screening questions on prescription drug use followed by questions on use of illegal substances. In the case of an affirmative response on the screening questions, a list of specific substances together with their "street-names" is presented. The list includes cocaine, amphetamines, and psychedelics, and five other classes of illegal substances (e.g. opioids, cannabis) as well as categories for "other" illegal substances and polysubstance use. Following this probe for the type of substance(s) used, questions are posed regarding the frequency and 
quantity. If a respondent reports using any illegal substance 5 or more times, questions for each substance of these substances are posed in order to assess symptoms of DSM-IV abuse and dependence. The section is skipped for respondents who would not answer openly to questions about illegal substance use. Inter-rater reliability of the CIDI substance use, abuse and dependence sections is in the acceptable range (kappa 0.55-0.83 for DSM-IV diagnoses of abuse or dependence of a substance) and good agreement was found between clinician-assigned DSMIV substance use diagnoses and those assigned according to the M-CIDI DSM-IV algorithms (kappa $0.83-0.86$ for DSM-IV diagnoses) $(33,35)$. In this study we refer to the lifetime use of cocaine, amphetamines and psychedelics five or more times.

\subsubsection{Assessment of psychotic symptoms}

Psychotic symptoms were assessed in the Psychosis section (G) of the M-CIDI/DIA-X at 4-year follow-up and 10-year follow-up. At the 4-year follow-up, prior lifetime history of psychotic symptoms was assessed, whereas at 10-year follow-up, symptoms during the interval period were assessed. A positive rating on any of the 15 core psychosis items of the M-CIDI (delusions, hallucinations) was regarded as a presence of a psychotic symptom. Participants were questioned to ensure that none of the positive responses were due to direct effects of medication, drug or alcohol use. The M-CIDI has been demonstrated to have a very low positive predictive value (0.226), a low specificity (0.6), a good negative predictive value (0.973) and sensitivity (0.875) for any specific psychotic disorder, such as schizophrenia (33). For this reason, psychotic symptoms, and not disorders, were assessed. This methodology has been utilized previously in epidemiologic work that has focused on examining the link between cannabis and psychosis (9). 


\subsection{Statistical analysis}

Data were weighted to consider different sampling probabilities as well as systematic non-response at baseline; numbers $(\mathrm{N})$ are reported unweighted. The Stata 10.1 software package was used to calculate proportions and standard errors as well as robust confidence intervals for weighted data (44). Cumulated data from baseline and all follow-up assessments are reported in this study. Since the follow-up CIDI questions refer to the time since the last interview, aggregating the information from the different waves reflects the cumulative lifetime status up to the last completed assessment at 10-year follow-up (either 4-year or 10-year follow-up; the cohort aged 14-17 years at baseline was also assessed at 18 months).

3021 participants completed the baseline assessment. Of those, 2719 completed 4-year or 10-year follow-up, the interviews in which psychotic symptoms were assessed. 131 participants refused to respond openly to the questions about illegal substance use on at least one assessment and were excluded leaving a sample of 2588 participants for our analyses.

To examine associations between the three illegal drug groups and psychotic symptoms, multinomial logistic regression analyses were used, with psychotic symptoms as the outcome and use of cocaine, amphetamines and psychedelics as independent variables, where those using a specific drug five or more times were compared to those using that substance zero to four times. First we report odds ratios (OR) and 95\% confidence intervals (95\% CI) from unadjusted logistic regression analyses. Second, we report adjusted odds ratios (AOR). Analyses were adjusted in two stages, first, for age, sex, social class, and urbanicity (Adjusted 1), and subsequently further adjusted for alcohol use disorder, nicotine dependence, cannabis use, other drug use disorder, any mood or anxiety disorder, and childhood adversity (Adjusted 2). 
Childhood adversity included any qualifying trauma before the age of ten, death of a father or mother before the age of ten, separation or divorce of parents before the age of ten, or not growing up with biological parents for most of the time (all assessed at the baseline interview). Variables such as drug use disorders (2), mood or anxiety disorders $(45,46)$, urbanicity $(28)$ and childhood adversity (27) have been adjusted for in the analysis because they have been demonstrated to be associated with psychotic symptoms. 


\section{Results}

Table 1 provides the cumulative lifetime incidence estimates of cocaine, amphetamine or psychedelic use in the EDSP sample until the 10-year follow-up. Additionally, it shows the distribution of sociodemographic factors and the cumulative incidence of substance use and specified mental disorders. Of the 2588 participants included in our analysis (Table 1), 1319 (number unweighted; percentage weighted: 49.5\%) were male, and the mean age at last assessment was 27.1 years. The cumulative incidence of lifetime cocaine use (five times or more) at 10-year follow-up was 5.5\%, 6.3\% for amphetamines, and 3.2\% for psychedelics. The cumulative lifetime incidence for any non-cannabinoid illegal substance (combined group of cocaine, amphetamines or psychedelics) use was $8.5 \%$.

At the last follow-up, the cumulative lifetime incidence of experiencing two or more psychotic symptoms was $218(8.3 \%)$ for the total sample $(\mathrm{N}=2588)$. Among persons having used a substance five or more times, 32 (22.5\%) experienced two or more psychotic symptoms for cocaine use, 37 (21.3\%) for amphetamine use, 24 (28.2\%) for psychedelics, and 49 (20.6\%) for any non-cannabinoid illegal substance (combined group of cocaine, amphetamines or psychedelics) (Table 2). The cumulative lifetime incidence of two or more psychotic symptoms did not differ between males $(121,8.9 \%, 95 \%$ CI $7.35-10.73)$ and females $(97,7.7 \%, 95 \%$ CI $6.27-9.58)$.

As shown in Table 3, a strong association was found between lifetime use of a substance and two or more psychotic symptoms in comparison to those without any psychotic symptoms (ORs ranging between 3.54-5.12). Although adjusting for covariates decreased the size of the associations, the lifetime use of cocaine, psychedelics and use of any non-cannabinoid illegal substance (combined group of cocaine, amphetamine, psychedelics) remained significantly 
associated with lifetime occurrence of two or more psychotic symptoms (AORs between 1.942.37). The association between amphetamine use and two or more psychotic symptoms did not remain significant after adjusting for covariates. After adjusting for covariates, the associations between lifetime substance use and one psychotic symptom were not significant for any of the substances examined.

In order to investigate whether the association between psychotic symptoms and substance use was independent of having a mood or anxiety disorder, we additionally performed subgroup analyses with data from all participants who had not met criteria for a mood or anxiety disorder during their lifetime $(\mathrm{N}=1224)$. A strong association was found between lifetime use of a substance five or more times and two or more psychotic symptoms in comparison to those without psychotic symptoms (ORs ranging from 4.28-8.23, Table 4). However, after adjusting for covariates, the association between lifetime substance use and two or more psychotic symptoms was significant only for psychedelics (OR 3.56, 95\% CI 1.20-10.61). Within this subgroup, none of the associations between lifetime substance use and one psychotic symptom remained significant after adjusting for covariates. 


\section{Discussion}

The main finding of the present study was that in adolescents and young adults, use of cocaine, amphetamine and/or psychedelics, was associated with psychotic symptoms. These associations were significant when adjusted for variables known to increase risk for psychosis, and most associations also remained after adjustment for use of alcohol, nicotine, and cannabis. Our results extend the previous work $(12,15-17)$ by showing an association between both psychedelics and stimulants (cocaine and amphetamine), and psychotic symptoms among a community-based population while excluding substance-induced psychosis. Among the subgroup of people without a mood or anxiety disorder, the strength of associations between use of a substance and psychotic symptoms was similar for cocaine, amphetamine, and the combined group of substances, however, psychedelics showed a slightly stronger association that remained significant after adjusting for covariates.

\subsection{Strengths and Limitations}

Strengths of the study include a population-based sample not limited by selection bias associated with treatment-seeking samples, and measurement of symptoms based on a standardised interview conducted by psychologists. Although community studies like the EDSP study data have some advantages in many ways, measurement of the outcomes is limited to psychotic symptoms (30). Despite the remarkable prevalence of $8.5 \%$ for psychotic symptoms in this community survey, data could not be analysed for diagnostic outcomes such as schizophrenia, or another psychotic disorder, because the M-CIDI cannot validly assess psychotic disorders according to DSM-IV criteria (33). Being based only on psychotic symptoms the results should not be interpreted as a positive association with psychosis in the 
sense of diagnostic categories, as individual symptoms are more likely to occur than symptoms meeting a full set of criteria for a diagnosis of a disorder. The low prevalence of psychotic symptoms also limited our ability to perform a prospective analysis to examine whether a temporal relationship exists between substance use and psychotic symptoms. It should be noted that the methodology used in the present study has been previously utilised and critically discussed in several other examinations on this topic with regard to cannabis disorders $(6,9)$. Additionally, two other limitations have to be noted. First, findings are based on self-reported use patterns and symptoms, which require accurate reports of the amount of a substance as well as accurate reports on symptoms. As such, there is a possibility of recall bias, but the confidential interview method used may help to minimize recall errors and erroneous information. Our study data assessed lifetime rates of substance use in a prospective, longitudinal design, which might account for somewhat elevated rates of substance use in comparison to some other studies, however, other population-based surveys, such as the National Household Survey on Drug Abuse found comparable estimates of substance use disorders. Among 12-29 year olds, 12-month rates of alcohol abuse were $27.4 \%$ and $14.5 \%$ for men and women, respectively, and $12.1 \%$ and $6.6 \%$ for alcohol dependence for men and women, respectively (47). Second, this community sample comprises a relatively well-educated and economically stable urban population. Therefore, estimates may not be generalisable to other more demographically diverse populations, and replication of findings in other community-based samples is needed.

\subsection{Clinical Relevance/Possible Implications}


Several cases of adverse reactions to illegal substances, including death, have been reported, however, many individuals use them without harm, which may engender a false sense of security from adverse outcomes (48). Illegal substance use and substance use disorders occur at a high rate among the population, especially among adolescents and young adults $(3,17,38,41)$ and the co-occurrence between illegal substance use and psychosis is more prevalent than would be predicted by chance alone $(20,49)$. While the exact mechanisms underlying the occurrence of prolonged psychotic symptoms associated with non-cannabinoid substance use are not known, theories have been proposed. There is some data to support a theory that repeated exposure to a stimulant, such as cocaine or amphetamines, leads to changes in the central nervous system, akin to 'kindling', implying that stimulant use may cause a psychosis that would not have occurred in the absence of the stimulant (50). There is less research in the area of prolonged psychotic symptoms associated with psychedelic use, however, a model where use of psychedelics might be associated with accelerating or precipitating the onset of an illness that would have ultimately have developed in an individual, has been proposed (51).

Because of conflicting evidence showing that substance use may begin before or after the onset of psychotic symptoms, studies examining the biochemical interactions and possible genetic factors at play will be important in proving or disproving the etiologic theories proposed. Studies examining genetic factors have begun in the areas of cannabis-psychosis association with examination of the catechol-O-methyltransferase gene (52) and methamphetamine-psychosis association with examination of protein interacting with C kinase (PICK1) gene (53) and polymorphisms therein that may modulate clinical outcomes after exposure to those substances. Similar genetic links associated with development of psychosis in individuals using other noncannabis substances should be investigated, in addition to further elucidating non-biologic 
factors such as environmental influences. Ongoing advances in neuroimaging such as diffusion tensor imaging (54) may also prove valuable in identifying structural abnormalities in many psychiatric disorders and possibly abnormalities common to both psychotic and substance use disorders.

In conclusion, findings from this study contribute to a growing body of literature that suggests that use of non-cannabinoid drugs such as cocaine, amphetamines or psychedelics is associated with psychotic symptoms. While neither the causality nor the possible biochemical mechanisms underlying this association have yet been demonstrated, this nonetheless presents a significant public health concern and the potential to utilize public education to reduce drug use and cases of psychosis in young adults should be carefully considered. Nonetheless, there remains a further need for longitudinal data in order to clarify the temporal sequence and whether substance use is, in fact, a causal factor in experiencing psychotic symptoms. 


\section{Funding/support}

J. Sareen, MD, was supported by a Canadian Institutes of Health Research New Investigator Award (\#152348). This work is part of the Early Developmental Stages of Psychopathology (EDSP) Study. The EDSP study is funded by the German Federal Ministry of Education and Research (BMBF; project no. 01EB9405/6, 01EB9901/6, EB01016200, 01EB0140, and 01EB0440). Part of the field work and analyses were also additionally supported by grants of the Deutsche Forschungsgemeinschaft (DFG; project no. LA1148/1-1, WI2246/1-1, WI709/7-1, and WI709/8-1). Principal authors of the EDSP study are H.-U. Wittchen, PhD, and R. Lieb, PhD. The authors declare no conflicts of interest.

\section{Acknowledgements}

Kirsten von Sydow, PhD, Gabriele Lachner, PhD, Axel Perkonigg, PhD, Peter Schuster, PhD, Michael Höfler, PhD, Holger Sonntag, Dipl.-Psych., Tanja Brückl, PhD, Elzbieta Garczynski, Dipl.-Psych., Barbara Isensee, PhD, Agnes Nocon, Dipl.-Psych., Chris Nelson, PhD, Hildegard Pfister, Dipl.-Inf., Victoria Reed, PhD, Barbara Spiegel, Dipl.-Soz., Andrea Schreier, PhD, Ursula Wunderlich, PhD, Petra Zimmermann, PhD, Katja Beesdo, PhD, Antje Bittner, PhD, Dipl.-Psych. Silke Behrendt and Dipl.-Psych. Susanne Knappe are current or past core staff members of the EDSP group. Scientific advisors are Jules Angst, MD (Zurich), Jürgen Margraf, PhD (Basel), Günther Esser, PhD (Potsdam), Kathleen Merikangas, PhD (NIMH, Bethesda), Ron Kessler, PhD (Harvard, Boston) and Jim van Os, PhD (Maastricht). 


\section{References}

1. Lieb R, Schuetz CG, Pfister H, von Sydow K, Wittchen H-U. Mental disorders in ecstasy users: a prospective-longitudinal investigation. Drug Alcohol Depend 2002;68:195-207.

2. Compton WM, Thomas YF, Stinson FS, Grant BF. Prevalence, correlates, disability, and comorbidity of DSM-IV drug abuse and dependence in the United States. Arch Gen Psychiatry 2007;64:566-576.

3. Wittchen H-U, Frölich C, Behrendt S, et al. Cannabis use and cannabis use disorders and their relationship to mental disorders: a 10-year prospective-longitudinal community study in adolescents. Drug Alcohol Depend 2007;88(Suppl 1):60-70.

4. Tien AY, Anthony JC. Epidemiological analysis of alcohol and drug use as risk factors for psychotic experiences. J Nerv Ment Dis 1990;178:473-480.

5. Arsenault L, Cannon M, Poulton R, Murray R, Caspi A, Moffitt TE. Cannabis use in adolescence and risk for adult psychosis: longitudinal prospective study. BMJ 2002;325:12121213.

6. van Os J, Bak M, Hanssen M, Bijl RV, de Graaf R, Verdoux H. Cannabis use and psychosis: a longitudinal population-based study. Am J Epidemiol 2002;156:319-327.

7. Zammit S, Allebeck P, Aandreasson S et al. Self reported cannabis use as a risk factor for schizophrenia in Swedish conscripts of 1969: historical cohort study. BMJ 2002;325: doi:10.1136/bmj.325.7374.1199.

8. Fergusson DM, Horwood LJ, Ridder EM. Tests of causal linkages between cannabis use and psychotic symptoms. Addiction 2005;100:354-366.

9. Henquet C, Krabbendam L, Spauwen J, et al. Prospective cohort study of cannabis use, predisposition for psychosis, and psychotic symptoms in young people. BMJ 2005;330:11; doi:10.1136/bmj.38267.664086.63.

10. Wiles NJ, Zammit S, Bebbington P, Singleton N, Meltzer H, Lewis G. Self-reported psychotic symptoms in the general population. Results from the longitudinal study of the British National Psychiatric Morbidity Survey. Br J Psychiatry 2006;188:519-526.

11. McGrath J, Welham J, Scott J, et al. Association between cannabis use and psychosis-related outcomes using sibling pair analysis in a cohort of young adults. Arch Gen Psychiatry 2010; doi: 10.1001/archgenpsychiatry.2010.6.

12. Dalmau A, Bergman B, Brismar B. Psychotic disorders among inpatients with abuse of cannabis, amphetamine and opiates. Do dopaminergic stimulants facilitate psychiatric illness? Eur Psychiatry 1999;14:366-371. 
13. Ringen PA, Melle I, Birkenæs AB, et al. Illicit drug use in patients with psychotic disorders compared with that in the general population: a cross-sectional study. Acta Psychiatr Scand 2008;117:133-138.

14. Degenhardt L, Hall W. The association between psychosis and problematical drug use among Australian adults: findings from the National Survey of Mental Health and Well-Being. Psychol Med 2001;31:659-668.

15. Farrell M, Boys A, Bebbington $P$, et al. Psychosis and drug dependence: results from a national survey of prisoners. Br J Psychiatry 2002;181:393-398.

16. McKetin R, McLaren J, Lubman DI, Hides L. The prevalence of psychotic symptoms among methamphetamine users. Addiction 2006;101:1473-1478.

17. Barnett JH, Werners U, Secher SM, et al. Substance use in a population-based clinic sample of people with first-episode psychosis. Br J Psychiatry 2007;190:515-520.

18. Mc Lellan AT, Woody GE, O’Brien CP. Development of psychiatric illness in drug abusers. Possible role of drug preference. NEJM 1979;301:1310-1314.

19. Hambrecht M, Häfner H. Substance abuse and the onset of schizophrenia. Biol Psychiatry 1996;40:1155-1163.

20. Cantor-Graae E, Nordstrom LG, McNeil TF. Substance abuse in schizophrenia: a review of the literature and a study of correlates in Sweden. Schizophr Res 2001;48:69-82.

21. Ross HE, Glaser FB, Germanson T. The prevalence of psychiatric disorders in patients with alcohol and other drug problems. Arch Gen Psychiatry 1988;45:1023-1031.

22. Fischer JF, Cho AK. Chemical release of dopamine from striatal homogenates: Evidence for an exchange diffusion model. J Pharmacol Exp Ther 1979;208:203-209.

23. Dackis CA, Gold MS. New Concepts in Cocaine Addiction: The Dopamine Depletion Hypothesis. Neurosci Biobehav Rev 1985;9:469-477.

24. Lang UE, Puls I, Müller DJ, Strutz-Seebohm N, Gallinat J. Molecular mechanisms of schizophrenia. Cell Physiol Biochem 2007;20:687-702.

25. Nichols DE. Hallucinogens. Pharmacol Ther 2004;101:131-181.

26. Stahl SM. Stahl's Essential Psychopharmacology. $3^{\text {rd }}$ ed. New York: Cambridge University Press; 2008.

27. van Os J, Jones P, Sham P, Bebbington P, Murray RM. Risk factors for onset and persistence of psychosis. Soc Psychiatry Psychiatr Epidemiol 1998;33:596-605. 
28. van Os J, Hanssen M, Bijl R, Vollebergh W. Prevalence of psychotic disorder and community level of psychotic symptoms: an urban-rural comparison Arch Gen Psychiatry 2001;58:663-668.

29. Wittchen H-U, Perkonigg A, Lachner G, Nelson CB. Early Developmental Stages of Psychopathology Study (EDSP): Objectives and Design. Eur Addict Res 1998;4:18-27.

30. Lieb R, Isensee B, von Sydow K, Wittchen H-U. The early developmental stages of psychopathology study (EDSP): A methodological update. Eur Addict Res 2000;6:170-182.

31. Wittchen H-U, Pfister H. DIA-X Interviews: Manual Für Screeningverfahren und Interview: PC Programm zur Durchführung des Inrterviews (Längs-und Querschnittuntersuchung); Auswertungsprogramm. Frankfurt: 1997, Swets \& Zeitlinger.

32. American Psychiatric Association: Diagnostic and Statistical Manual of Mental Disorders. 4th ed. Washington, D.C.: American Psychiatric Association; 1994.

33. Reed V, Gander F, Pfister H, et al. To what degree the Composite International Diagnostic Interview (CIDI) correctly identifies DSM-IV disorders? Int J Methods Psychiatr Res 1998;7:142-155.

34. Wittchen H-U, Lachner G, Wunderlich U, Pfister H. Test-retest reliability of the computerized DSM-IV version of the Munich-Composite International Diagnostic Interview (MCIDI). Soc Psychiatry Psychiatr Epidemiol 1998;33:568-578.

35. Lachner G, Wittchen H-U, Perkonigg A, et al. Structure, content and reliability of MunichComposit International Diagnostic Interview (M-CIDI) substance use sections. Eur Addict Res 1998;4:28-41.

36. Lieb R, Pfister H, Wittchen H-U. Use, abuse and dependence of prescription drugs in adolescents and young adults. Eur Addict Res 1998;4:67-74.

37. Lieb R, Schreier A, Pfister H, Wittchen H-U. Maternal smoking and smoking in adolescents: a prospective community study of adolescents and their mothers. Eur Addict Res 2003;9:120130.

38. Perkonigg A, Pfister H, Höfler M, et al. Substance use and substance use disorders in a community sample of adolescents and young adults: incidence, age effects and patterns of use. Eur Addict Res 2006;12:187-196.

39. Behrendt S, Wittchen H-U, Höfler M, et al. Risk and speed of transitions to first alcohol dependence symptoms in adolescents: a 10-year longitudinal community study in Germany. Addiction 2008;103:1638-1647. 
40. Perkonigg A, Goodwin RD, Fiedler A, et al. The natural course of cannabis use, abuse, and dependence during the first decades of life. Addiction 2008;103:439-449.

41. Wittchen H-U, Behrendt S, Höfler M, et al. What are the high risk periods for incident substance use and transitions to abuse and dependence? Implications for early interventions and prevention. Int J Methods Psychiatr Res 2008;17(Suppl 1):16-29.

42. Behrendt S, Wittchen H-U, Höfler M, Lieb R, Beesdo K. Transitions from first substance use to substance use disorders in adolescence: Is early onset associated with a rapid escalation? Drug Alcohol Depend 2009;99:68-78.

43. Wittchen H-U, Behrendt S, Höfler M, et al. A typology of cannabis-related problems among individuals with repeated illegal drug use in the first three decades of life: Evidence for heterogeneity and different treatment needs. Drug Alcohol Depend 2009;102:151-157.

44. STATACORP. STATA Statistical Software. Release 10.1 ed. Texas: College Station, 2009.

45. Koreen AR, Siris SG, Chakos M, Alvir J, Maverhoff D, Lieberman J. Depression in firstepisode schizophrenia. Am J Psychiatry 1993;150:1643-1648.

46. Michail M, Birchwood M. Social anxiety disorder in first-episode psychosis: incidence, phenomenology and relationship with paranoia. Br J Psychiatry 2009;195:234-241.

47. Harford TC, Grant BF, Yi HY, Chen CM. Patterns of DSM-IV alcohol abuse and dependence criteria among adolescents and adults: results from the 2001 National Household Survey on Drug Abuse. Alcohol Clin Exp Res 2005;29:810-828.

48. Burgess C, O’Donohoe A, Gill M. Agony and ecstasy: a review of MDMA effects and toxicity. Eur Psychiatry 2000;15:287-294.

49. Regier DA, Farmaer ME, Rae DS, et al. Comorbidity of mental disorders with alcohol and other drug abuse. Results from the Epidemiologic Catchment Area (ECA) Study. JAMA 1990;264:2511-2518.

50. Curran C, Byrappa N, McBride A. Stimulant psychosis: systematic review. Br J Psychiatry 2004;185:196-204.

51. Strassman RJ. Adverse reactions to psychedelic drugs. A review of the literature. J Nerv Ment Dis 1984;172:577-595.

52. Caspi A, Moffitt TE, Cannon M, et al. Moderation of the effect of adolescent-onset cannabis use on adult psychosis by a functional polymorphism in the cathecol-O-methyltransferase gene: Longitudinal evidence of a gene X environment interaction. Biol Psychiatry 2005;57:11171127. 
53. Matsuzawa D, Hashimoto K, Miyatake R, et al. Identification of functional polymorphisms in the promoter region of the human PICK1 gene and their association with methamphetamine psychosis. Am J Psychiatry 2007;164:1105-1114.

54. White T, Schmidt M, Karatekin C. White matter 'potholes' in early-onset schizophrenia: A new approach to evaluate white matter microstructure using diffusion tensor imaging. Psychiatry Res 2009;174:110-115. 